\title{
Diagnostic Accuracy of WINROP, CHOP-ROP and ROPScore in Detecting Type 1 Retinopathy of Prematurity
}

\author{
Deena Thomas, ${ }^{1}$ Shamnad Madathil, ${ }^{1}$ Anu Thukral, ${ }^{1}$ M Jeeva Sankar, ${ }^{1}$ Parijat Chandra, ${ }^{2}$ \\ RAMESh Agarwal, ${ }^{1}$ ASHOK DeORARI ${ }^{1}$ \\ From ${ }^{1}$ Division of Neonatology, Department of Pediatrics, All India Institute of Medical Sciences; and ${ }^{2}$ Department of \\ Ophthalmology, Dr. Rajendra Prasad Center for Ophthalmic Sciences; New Delhi. \\ Correspondence to: Dr Anu Thukral, Associate Professor, Department of Pediatrics, All India Institute of Medical Sciences, \\ New Delhi 110029.dranuthukral@gmail.com \\ Received: November 06, 2020; Initial review: December 27, 2020; Accepted: May 12, 2021.
}

Background: Algorithms for predicting retinopathy of prematurity (ROP) requiring treatment need to be validated in Indian settings to determine if the burden of screening can be reduced without compromising the sensitivity of existing gestation and weight-based cut offs.

Objective: To evaluate the performance of the available algorithms namely, WINROP (Weight, Insulin-like growth factor I, Neonatal ROP), CHOP-ROP (Children's Hospital of Philadelphia $\mathrm{ROP}$ ) and ROPScore in predicting type $1 \mathrm{ROP}$ and time from alarm to treatment by each algorithm.

Study design: Ambispective observational.

Setting: Tertiary care neonatal intensive care unit in India.

Participants: Neonates less than 32 weeks or less than $1500 \mathrm{~g}$ born between July, 2013 to June, 2019 ( $N=578)$, who underwent ROP screening.

Primary outcome: Sensitivity, specificity and time from alarm to treatment by each algorithm.

Results: The sensitivity and specificity of WINROP was $85 \%$ and $36 \%$, for CHOP-ROP it was $54 \%$ and $71 \%$, and for ROPScore it was $73 \%$ and $67 \%$, respectively in detecting type 1 ROP. A total of $50 / 51$ (98\%) of neonates with type 1 ROP underwent treatment at median gestation of 9 weeks and median time from alarm to treatment by WINROP, CHOP-ROP and ROPScore was 7, 7 and 3 weeks, respectively.

Conclusion: WINROP, CHOP-ROP and ROPScore were not sensitive enough to replace the gestational age, weight and risk factor-based screening criteria for type 1 ROP.

Keywords: Neonatal intensive care unit, Premature, Sensitivity, Specificity.

Published online: May 20, 2021; PII: S097475591600328

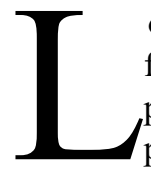

ow- and middle-income countries are currently facing the third epidemic of retinopathy of prematurity (ROP) on account of higher rate of preterm birth and wide variations in neonatal care provided. Blencowe, et al. [1] estimated that approximately 98077 neonates in India would require screening for ROP amounting to nearly three lakh examinations every year. National guidelines recommend screening of all the neonates $<34$ weeks or $<2000$ gram or neonates with gestational age between 34-36 weeks with risk factors for ROP such as prolonged oxygen support, cardiovascular instability, and sepsis [2]. When compared to screening criteria in developed countries, these guidelines are much higher, as bigger babies also develop severe ROP in developing countries, and this further increases the screening load [3,4]. Given the paucity of skilled ophthalmologists for screening; gestation and weightbased screening criteria increase the burden on existing health systems, leading to poor quality of services being provided and eventually leading to missing out on cases requiring close follow up and treatment.
Current conventional screening method for ROP is a painful procedure. It leads to physiological changes like hypertension and decrease in oxygen saturation [5]. In addition, this is an additional burden on the fragile health system. Many screening algorithms have been developed and are in place for more than a decade now. However, due to their inability in providing 100\% sensitivity (assuming gestation and weight risk factorbased screening criteria as standard), none of the algorithms have been able to replace existing protocols. These algorithms have shown high sensitivity and negative predictive value in many countries; however, they have not been widely validated in Indian settings [68]. Due to lack of sufficient literature in Indian settings, this study was planned with the aim to evaluate the diagnostic performance of all the three algorithms, namely WINROP (Weight, Insulin-like growth factor I, Neonatal ROP), CHOP-ROP (Children's Hospital of Philadelphia ROP) and ROPScore in predicting type 1 ROP in an Indian setting $[9,10]$. We also evaluated time from alarm to treatment by each algorithm. 


\section{METHODS}

This study was conducted as an ambispective observational study with a retrospective phase collecting data from 1 July, 2013 to 30 June, 2018 and a prospective phase comprising of data collected from 1 July, 2018 to 30 June, 2019 at a tertiary care hospital. The policy of our unit is to screen all neonates less than 32 weeks gestational age (GA) or neonates with a birthweight less than $1500 \mathrm{~g}$ or bigger neonates (32-34 weeks GA or bithweight 1501-2000 g) with risk factors (respiratory or hemodynamic instability, anemia requiring transfusion or culture positive sepsis). Our unit has a strict pulse oximetry monitoring policy for preterm infants care in the NICU. Since only neonates less than 32 weeks GA can be entered in WINROP and ROPScore, the neonates less than 32 weeks or birthweight less than $1500 \mathrm{~g}$ who underwent retinopathy of prematurity screening were included in the study. Neonates with congenital malformation, hydrocephalus and hydrops fetalis were excluded.

Records of all the neonates who underwent ROP screening in the retrospective phase were retrieved from ROP registers maintained in the unit. In addition, all the demographic details, and antenatal, intrapartum and postnatal course details were retrieved from the medical records department. Birthweight, gestational age and weekly weight (weight on postnatal day 8, 15, 22, 29 and so on) of these infants till discharge was noted. Neonates on invasive ventilation were weighed on alternate days after disconnecting from ventilator for a brief duration as per the unit policy. The appropriateness of birthweight for gestational age was assigned by the AIIMS intrauterine growth chart [11] for neonates $\geq 32$ weeks of gestation or Lubchenco growth charts [12] for neonates less than 32 weeks of gestation.

All the infants satisfying the inclusion criteria were screened for ROP as per the unit protocol at 4 weeks of postnatal age with the exception of those $<28$ weeks whose first screen was done at 2-3 weeks postnatal age. ROP was described as per International Classification of Retinopathy of Prematurity and was classified into treatment group as per Early Treatment of Retinopathy of Prematurity Classification [13,14]. The worst stage of ROP and the presence of plus disease (when present) was recorded. In cases where both eyes were affected, worst stage of the ROP of either eye was taken. Postnatal age of development of type 1 ROP as defined by any ROP in Zone I with plus disease or stage 3 ROP in zone I without plus disease or stage 2 or 3 ROP in Zone II with plus disease was noted and the treatment provided was also recorded. The infants with type 1 ROP findings who were lost to follow up were contacted telephonically to know their ophthalmological outcome and intervention done (laser photocoagulation/anti-VEGF injection). Similar data collection was performed for the prospective phase after informed parental consent. Ethical clearance was obtained from institute's ethics committee.

Data obtained from included neonates was entered into the following three predictive algorithms according to the eligibility criteria:

WINROP: All the neonates less than 32 weeks of gestation at birth irrespective of the BW were eligible to be entered into WINROP, which is available online (www.winrop.com) [15]. Birthweight, gestational age and weekly weight were entered till 35 weeks of postmenstrual age or discharge, or till the alarm signals in the algorithm, whichever was earlier. WINROP algorithm requires that the weight of neonate be entered till 35 weeks of postmenstrual age (PMA) to classify a neonate to be at low risk.

CHOP-ROP: Neonates less than 31 weeks of GA or less than $1501 \mathrm{~g}$ birthweight were eligible to be evaluated by CHOP-ROP [16]. Birthweight, gestational age and daily weight gain rate was entered into the algorithm to calculate the risk score from 2 nd week onwards. CHOPROP requires documentation of neonatal weight at end of second week to be included in the algorithm. Weight change in the first week was disregarded as per the original study. Daily weight gain rate was calculated by weekly measurements (difference between current weight and previous week's weight divided by 7). For neonates with gestation $>28$ week, only birth weight and weight gain rate was used for calculation. Alarm cutoff of $\geq 0.010$ was used to identify neonates at risk of type 1 ROP.

ROP score: Neonates less than 32 weeks or $<1500 \mathrm{~g}$ whose weight at end of 6 th week postnatal age was available before discharge or at follow up were eligible to be included in the ROPScore algorithm proposed by Eckert, et al. [17]. This score required data on use of oxygen in mechanical ventilation (invasive or non-invasive ventilation including CPAP upto sixth completed week), requirement of blood transfusion up to sixth completed week of life, neonate's weight at sixth completed week in addition to birthweight and gestational age: ROPScore excel sheet was used for calculation of the score. Cutoff for risk of type 1 ROP was taken as $\geq 14.5$.

Primary outcomes were to evaluate the specificity and the sensitivity of three screening algorithms namely, WINROP, CHOP-ROP and ROPScore, in predicting type 1 ROP. Secondary outcome was time from alarm to predict type 1 ROP by these algorithms to the time the neonates underwent treatment for the same. 
The reported specificity for CHOP-ROP was $51 \%$, for ROPScore $57 \%$, and for WINROP was $60 \%[6-9,18]$. To detect a similar magnitude of difference (i.e. absolute difference of 9\%) between CHOP-ROP and WINROP algorithms, with a power of $80 \%$ and alpha error of $5 \%$, a total of 473 neonates had to be enrolled.

Statistical analysis: Statistical analysis was done using Stata 12.0 (StataCorp). Diagnostic performance of all the three algorithms was described by calculating sensitivity, specificity, positive predictive value, negative predictive value, positive likelihood ratio, negative likelihood ratio along with $95 \%$ confidence interval for predicting the risk of type 1 ROP using Open Epi ver 3.01. The receiver operating characteristics (ROC) curve was constructed, and the cutoff of ROPScore and CHOP-ROP with 100\% sensitivity and maximal specificity was calculated.

\section{RESULTS}

Out of 15,405 neonates born during the study period with 898 neonates were less than 32 weeks GA or birth weight $<1500 \mathrm{~g}$. The records of 578 neonates who underwent at least one ROP screening satisfying the inclusion criteria were available. A total of 382 out of $578(66 \%), 498$ out of $578(86 \%)$ and 370 out of $578(64 \%)$ neonates could be analyzed for their risk of developing type 1 ROP using WINROP, CHOP-ROP and ROPScore algorithms, respectively. Fig. 1 describes the study flow and reasons for exclusion from the study.

Neonates included in the study had a mean (SD) GA and birth weight of 30.3 (2.4) weeks and 1184 (308) gms, respectively. Other demographic details have been provided in Table I. One third of the neonates were noted to have any ROP with a quarter of them requiring treatment

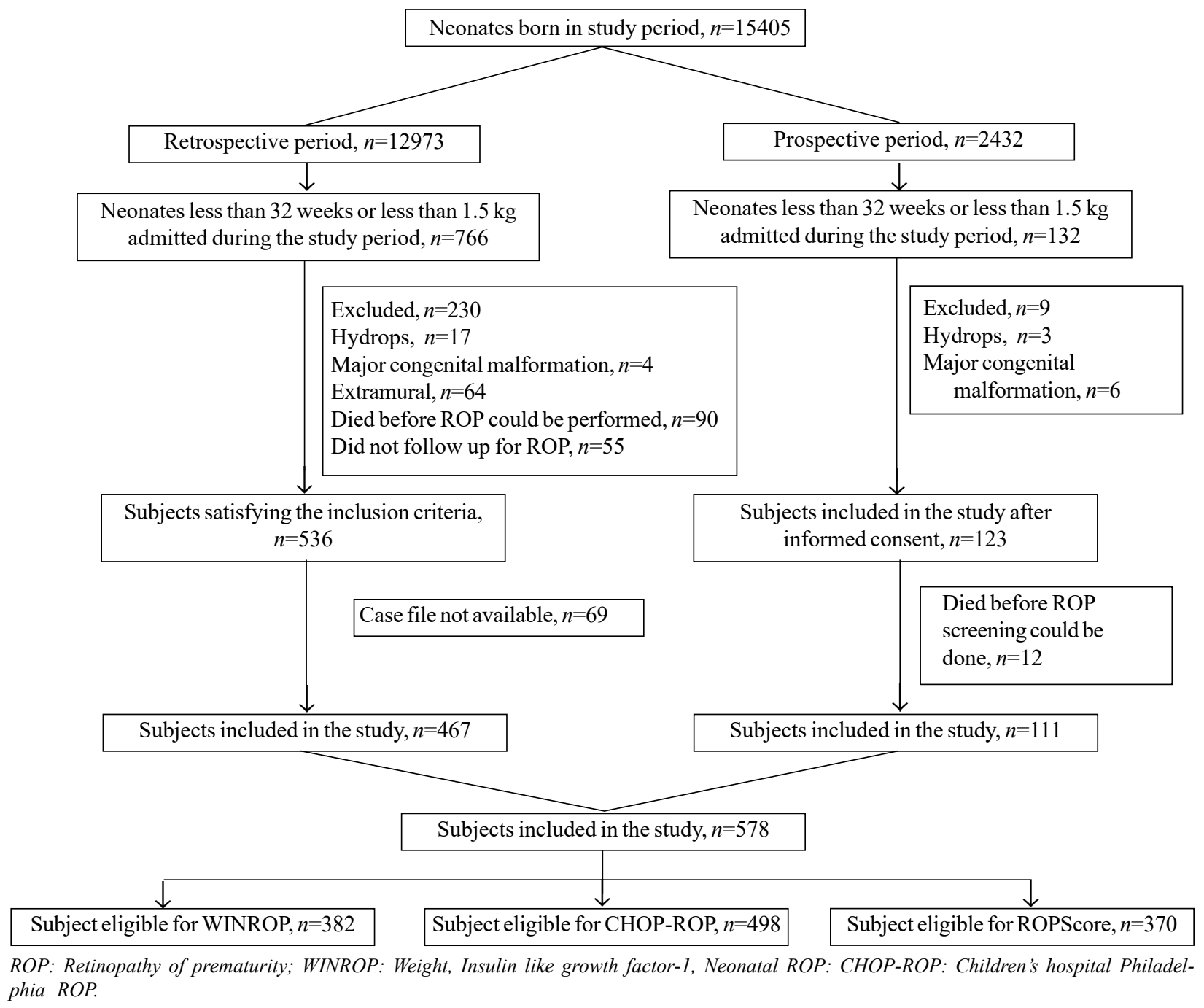

Fig. 1 Study flow. 
Table I Baseline Characteristics of the Study Population $(N=578)$

\begin{tabular}{|c|c|}
\hline Characteristics & Value \\
\hline Gestational age (wk) ${ }^{a}$ & $30.3(2.37)$ \\
\hline Birthweight $(\mathrm{g})^{a}$ & $1184(308)$ \\
\hline Small for gestational age & $234(40.5)$ \\
\hline Male & $306(52.9)$ \\
\hline Singleton & $414(71.6)$ \\
\hline Complete antenatal steroid coverage & $350(60.5)$ \\
\hline Resuscitation (more than initial steps) & $181(31.3)$ \\
\hline Apgar score at $1 \min ^{a}$ & $6.1(2.02)$ \\
\hline Apgar score at $5 \mathrm{~min}^{a}$ & $7.5(1.3)$ \\
\hline Respiratory distress requiring surfactant & $176(30.4)$ \\
\hline Bronchopulmonary dysplasia & $81(14)$ \\
\hline Invasive ventilation & $150(26)$ \\
\hline Invasive ventilation duration $(\mathrm{d})^{b, c}$ & $6(3-19)$ \\
\hline Grade III or IV intraventricular hemorrhage ${ }^{d}$ & $18(3.2)$ \\
\hline Periventricular leukomalacia ${ }^{d}$ & $67(11.6)$ \\
\hline Hemodynamically significant ductus arteriosus & $61(10.5)$ \\
\hline Hypotension requiring inotropes & $60(10.4)$ \\
\hline Sepsis requiring antibiotics & $182(31.4)$ \\
\hline Day of regaining birth weight ${ }^{a}$ & $11.9(5.3)$ \\
\hline Anemia requiring transfusion & $112(19.4)$ \\
\hline
\end{tabular}

(Table II). No neonate less than 32 weeks having type 1 ROP was missed by the existing screening protocol; amounting to sensitivity of $100 \%$ in this age group. Around $70(12 \%)$ neonates were lost to follow up from the screening protocol out of which 5 neonates had type 1 or 2 ROP on last screen available and were contacted telephonically to know their final ophthalmological outcome. All but one neonate with type 1 ROP underwent treatment for the same at a median postnatal age of 9 weeks or 36 weeks postmenstrual age. Only one baby received anti- VEGF injection during the study period.

Diagnostic performance of the three screening algorithms has been provided in Table III. WINROP had the maximum sensitivity $(85 \%)$ to identify neonates with type 1 ROP followed by ROPScore and then CHOP-ROP. Specificity followed the reverse order with CHOP-ROP being most specific (71\%). Decreasing the cutoff point of ROPScore to 10.79 gave $100 \%$ sensitivity with a specificity of $16.5 \%(12.8 \%-20.9 \%)$ and avoided screening in 61 neonates. WINROP and CHOP-ROP identified type 1 ROP earliest at 2 weeks of postnatal age, around 7 weeks before conventional screening method where the neonates with type 1 ROP were identified and treated at 9 weeks of
Table II Retinopathy of Prematurity in the Study Population

\begin{tabular}{|c|c|c|c|}
\hline Characteristics & $\begin{array}{l}\text { Retrospective } \\
\text { Cohort } \\
(n=467)\end{array}$ & $\begin{array}{l}\text { Prospective } \\
\text { Cohort } \\
(N=111)\end{array}$ & $\begin{array}{r}\text { Combined } \\
(n=578)\end{array}$ \\
\hline Any ROP & $183(39.2)$ & $25(22.5)$ & $208(36)$ \\
\hline \multicolumn{4}{|l|}{ Type of ROP } \\
\hline Type 1 & $42(8.9)$ & $9(8.1)$ & $51(8.8)$ \\
\hline Type 2 & $18(3.8)$ & $1(0.9)$ & $19(3.3)$ \\
\hline Mild ROP & $123(26.3)$ & $15(13.5)$ & $138(23.9)$ \\
\hline $\begin{array}{l}\text { Identification of } \\
\text { any ROP (wk) }{ }^{a, b}\end{array}$ & $6(4-8)$ & $7(6-9)$ & $6(4-8)$ \\
\hline $\begin{array}{l}\text { Identification of type1 } \\
\text { ROP (wk) }{ }^{a, b}\end{array}$ & $9(7-10)$ & $9(7-12)$ & $9(7-10)$ \\
\hline Number of screenings ${ }^{a}$ & $3(2-5)$ & $3(2-4)$ & $3(2-5)$ \\
\hline
\end{tabular}

postnatal age. ROPScore identified neonates at risk of type of type 1 ROP at 6 weeks of postnatal age, by which time 3 neonates were already treated for type 1 ROP by conventional screening method. ROC curve of CHOPROP and ROPScore for identifying type 1 ROP among 334 neonates showed area under curve of ROPScore [0.75 (0.66-0.83)] to be more than that of CHOP-ROP [0.66 (0.580.95)] (Fig. 2). Since WINROP gives only binary output to signify the risk of developing type 1 ROP unlike a continuum of scores provided by CHOP-ROP and ROPScore, an ROC curve for the same was not constructed.

\section{DISCUSSION}

The study was conducted at a level III neonatal intensive care unit on intramural neonates. The unit caters mainly to high risk neonates who are referred in utero from many parts of North India early in gestation and where gentle ventilation guided by pulse oximetry along with antibiotic stewardship is the norm.

Our rates of ROP and type I ROP were higher than the literature [19], possibly due to the smaller gestational age and lesser birthweight of our neonates. Sensitivity of WINROP in our cohort was $85.42 \%$ which was slightly lower than the recent study by Sanghi, et al. [10] (90\%). Low sensitivity (65\%) of WINROP was observed in a study in Taiwan where older and larger neonates developed ROP requiring treatment which were missed by the WINROP [20]. The specificity (36\%), positive predictive value (16\%) and high negative predictive value $(94 \%)$ in our study was in accordance with the previously reported literature $[8,21,22]$.

CHOP-ROP performed poorly in our cohort with a sensitivity of $54 \%$. This was lower than that reported by 
Table III Diagnostic Performance of WINROP, CHOP-ROP and ROPScore

\begin{tabular}{lllr}
\hline Parameter & $\begin{array}{l}\text { WINROP } \\
(n=382)\end{array}$ & $\begin{array}{l}\text { CHOP-ROP } \\
(n=498)\end{array}$ & $\begin{array}{r}\text { ROPScore } \\
(n=370)\end{array}$ \\
\hline Sensitivity (\%) & 85.4 & 54 & 72.9 \\
& $(72.8-92.7)$ & $(40.4-67.0)$ & $(59-83.4)$ \\
Specificity (\%) & 36.2 & 71.4 & 67.3 \\
& $(31.3-41.5)$ & $(67.1-75.4)$ & $(61.9-2.2)$ \\
PPV (\%) & 16.1 & 17.4 & 25 \\
& $(12.1-21.2)$ & $(12.3-24.2)$ & $(18.6-32.8)$ \\
NPV (\%) & 94.5 & 93.3 & 94.3 \\
& $(89.1-97.3)$ & $(90.1-95.5)$ & $(90.5-96.6)$ \\
Positive LR & 1.3 & 1.9 & 2.3 \\
& $(1.3-1.4)$ & $(1.7-2.0)$ & $(2.1-2.3)$ \\
Negative LR & 0.4 & 0.6 & 0.4 \\
& $(0.3-0.5)$ & $(0.6-0.7)$ & $(0.3-0.5)$ \\
Diagnostic OR & 3.3 & 2.9 & 5.5 \\
& $(1.4-7.6)$ & $(1.6-5.3)$ & $(2.8-10.9)$ \\
NNS & 9.4 & 9.6 & 5.2 \\
& $(5.9-21.4)$ & $(6.2-21.1)$ & $(3.8-7.9)$ \\
\hline
\end{tabular}

95\% CI in parenthesis. ROP-retinopathy of prematurity; WINROPweight, insulin-like growth factor I, neonatal, ROP; CHOP-ROPChildren's Hospital of Philadelphia ROP; PPV-Positive predictive value; NPV-Negative predictive value; LR-likelihood ratio; ORodds ratio; NNS-Number needed to screen.

Doshi, et al. [9] (67\%) in 2019 Indian infants in spite of their cohort dealing with bigger neonates. They used the nomogram provided by Binenbaum, et al. [16] for manual calculation of alarm limit. This method was not considered feasible in our setting due to large sample size and hence the original formula provided by Binenbaum, et al. [16] was used. In the study by Doshi, et al. [9] decreasing the cutoff from 0.014 to 0.010 gave $100 \%$ sensitivity. However, in our study the cutoff had to be decreased to 0.001 to give $100 \%$ sensitivity, which in turn decreased the specificity to unacceptable levels (2.23\%).

The sensitivity of ROPScore was $73 \%$ which was lower than previous studies $(95-100 \%)[6,23]$. When the cutoff of ROPScore was decreased to 10.79 , the sensitivity approached $100 \%$ and this cut off potentially would avoid screening in $16.5 \%$ of neonates and thus has clinical implication. ROPScore showed better diagnostic performance with an area under curve of 0.75 vs 0.66 of CHOP-ROP. However, ROPScore has inherent disadvantages as it gives an alarm at 6 weeks of postnatal age when most of the neonates with aggressive posterior ROP are already identified by conventional screening methods and treated. In addition, many neonates with risk factors who are discharged before six weeks of postnatal age cannot be evaluated using ROPScore thereby missing out on cases with type 1 ROP.

INDIAN PEDIATRICS
The median time from alarm to treatment in our study for WINROP, CHOP-ROP and ROPScore was 7, 7 and 3 weeks, respectively which was lower than those previously estimated [24], where it was 11.1, 9.1 and 5.1 week, respectively.

An ideal algorithm for identifying type $1 \mathrm{ROP}$ is the one with $100 \%$ sensitivity and a reasonable level of specificity so as to reduce the unwanted ROP screenings being done currently. None of the algorithms were sensitive enough in our setting probably due to a higher saturation target of $90-95 \%$ being followed in the unit. A similar decrease in sensitivity of WINROP from $87.5 \%$ to $48 \%$ was noted by Lundgren, et al. [25] when the saturation targets increased from $88-92 \%$ in $2011-2012$ to $91-95 \%$ in 2015-2016.

Strengths of our study are its large sample size, and using registers maintained by the staff and doctors of the unit containing data of neonates who underwent ROP screening to retrieve the files of neonates who underwent screening, and this was cross-checked with the electronic discharge data of the unit. Three rounds of file retrieval from medical records department was conducted before classifying a file as non-available. Our study has some limitations as well. The weight was not available at 6 weeks completed age in 196 out of 467 (42\%) neonates enrolled in retrospective phase. None of the algorithms could accommodate all the neonates included in the study, thereby true comparison of diagnostic performance of the various algorithms with the existing weight and gestationbased criteria could not be performed.

In conclusion, none of the screening algorithms with their recommended cutoffs was able to provide $100 \%$ sensitivity as provided by the weight, gestational age and

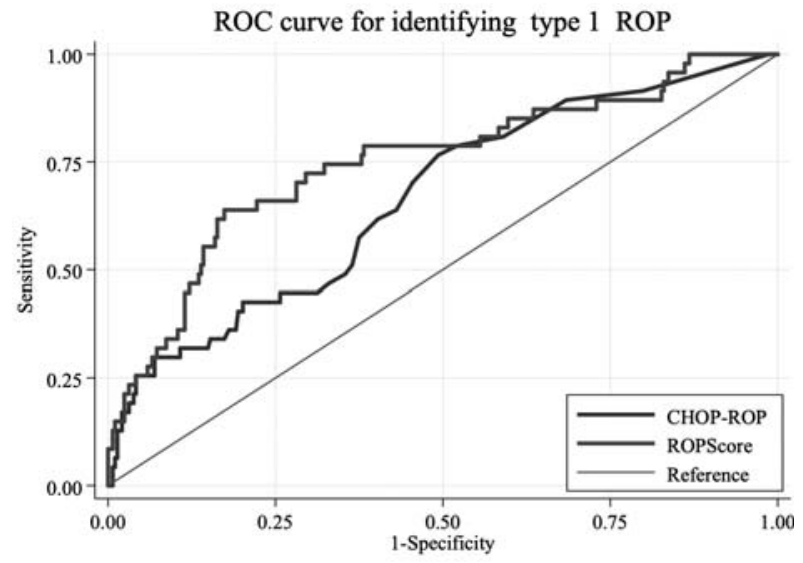

ROC: Receiver operating characteristics curve; CHOP-ROP-Children's Hospital of Philadelphia ROP; ROP-retinopathy of prematurity.

Fig. 2 ROC curve of CHOP-ROP and ROPScore for identifying type 1 ROP. 


\section{WHAT IS ALREADY KNOWN?}

- Gestational age, weight based as well as risk factor-based criteria are generally followed to screen neonates at risk for developing type 1 ROP.

\section{WHAT THIS STUDY ADDS?}

- None of the three screening algorithms examined in the study was able to provide $100 \%$ sensitivity as provided by the weight, gestational age and risk factor-based screening protocol.

risk factor-based screening protocol being currently followed in the unit. Although ROPScore with a modified cutoff of 10.79 looks promising since it has $100 \%$ sensitivity, it has a poor specificity of $16.5 \%$ and it gives an alarm at 6 weeks completed age, a time at which few of the neonates would already have been identified by conventional screening method.

Ethics clearance: Institutional ethics committee of Post Graduate research (clinical sciences), AIIMS, New Delhi; No. IECPG-280 dated 28 June, 2018.

Contributors: DT: prepared the first draft of the protocol and had the prime responsibility of data collection, data analysis and compilation of results; SM: collected data, cross checked data entry and contributed to the manuscript; AT: conceptualized the study, supervised data entry and provided input in preparation of protocol and final manuscript; MJS: contributed to protocol formation, helped in statistical analysis and contributed to final manuscript; PC: valuable suggestion during protocol formation and provided input to final manuscript; RA: critically reviewed the protocol of the study, ensured timely progress of the study via departmental meetings and provided input to final manuscript; AD: input in protocol of the study and critically reviewed the final manuscript. All the authors in principal agreed to the final manuscript of the study.

Funding: None; Competing interest: None stated.

\section{REFERENCES}

1. Blencowe H, Moxon S, Gilbert C. Update on blindness due to retinopathy of prematurity globally and in India. Indian Pediatr. 2016;53:S89-92.

2. Shukla R, Murthy GVS, Gilbert C, et al. Operational guidelines for ROP in India: A Summary. Indian J Ophthalmol. 2020;68:S108-14.

3. Fierson WM; American Academy of Pediatrics Section on Ophthalmology; American Academy of Ophthalmology; American Association for Pediatric Ophthalmology and Strabismus; American Association of Certified Orthoptists. Screening Examination of Premature Infants for Retinopathy of Prematurity. Pediatrics. 2018;142: e20183061.

4. Shah PK, Narendran V, Kalpana N, et al. Severe retinopathy of prematurity in big babies in India: History repeating itself? Indian J Pediatr. 2009;76:801-4.

5. Jiang J-B, Zhang Z-W, Zhang J-W, et al. Systemic changes and adverse effects induced by retinopathy of prematurity screening. Int J Ophthalmol. 2016;9:1148-55.

6. Cagliari PZ, Lucas VC, Borba IC, et al. Validation of ROPScore to predict retinopathy of prematurity among very low birth weight preterm infants in a southern Brazilian population. Arq Bras Oftalmol. 2019;82:476-80.

7. Timkovic J, Pokryvkova M, Janurova K, et al. Evaluation of the WinROP system for identifying retinopathy of prematurity in Czech preterm infants. Biomed Pap Med Fac Univ Palacky Olomouc Czechoslov. 2017;161:111-6.

8. Jung JL, Wagner BD, McCourt EA et al. Validation of WINROP for detecting retinopathy of prematurity in a North American cohort of preterm infants. J AAPOS. 2017;21:229-233.

9. Doshi S, Desai S, Nanavati R, et al. Children's hospital of Philadelphia Score to predict severe retinopathy in Indian preterm infants. Eye. 2019;33:1452-8.

10. Sanghi G, Narang A, Narula S, et al. WINROP algorithm for prediction of sight threatening retinopathy of prematurity: Initial experience in Indian preterm infants. Indian $\mathbf{J}$ Ophthalmol. 2018;66:110-3.

11. Singh M, Giri SK, Ramachandran K. Intrauterine growth curves of live born single babies. Indian Pediatr. 1974;11:475-9.

12. Lubchenco LO, Hansman C, Dressler M, Boyd E. Intrauterine growth as estimated from liveborn birth-weight data at 24 to 42 weeks of gestation. Pediatrics. 1963; 32:793.

13. International Committee for the Classification of Retinopathy of Prematurity. The International Classification of Retinopathy of Prematurity revisited. Arch Ophthalmol. 2005;123:991-9.

14. Hardy RJ, Good WV, Dobson V, et al. The early treatment for retinopathy of prematurity clinical trial: Presentation by subgroups versus analysis within subgroups. $\mathrm{Br} \mathrm{J}$ Ophthalmol. 2006;90:1341-2.

15. Löfqvist C, Andersson E, Sigurdsson J, et al. Longitudinal postnatal weight and insulin-like growth factor 1 measurements in the prediction of retinopathy of prematurity. Arch Ophthalmol. 2006;124:1711-8.

16. Binenbaum G, Ying G, Quinn GE, et al. The CHOP postnatal weight gain, birth weight, and gestational age retinopathy of prematurity risk model. Arch Ophthalmol. 2012;130: 1560-5.

17. Eckert GU, Fortes Filho JB, Maia M, et al. A predictive score for retinopathy of prematurity in very low birth weight preterm infants. Eye. 2012;26:400-6.

18. Ali E, Al-Shafouri N, Hussain A, et al. Assessment of WINROP algorithm as screening tool for preterm infants in Manitoba to detect retinopathy of prematurity. Paediatr Child Health. 2017;22:203-6.

19. Kumar P, Sankar MJ, Deorari A, et al. Risk factors for severe retinopathy of prematurity in preterm low birth weight neonates. Indian J Pediatr. 2011;78:812-6. 
20. Ko C-H, Kuo H-K, Chen C-C, et al. Using WINROP as an adjuvant screening tool for retinopathy of prematurity in Southern Taiwan. Am J Perinatol. 2015;30:149-54.

21. Sun H, Kang W, Cheng X, et al. The use of the WINROP screening algorithm for the prediction of retinopathy of prematurity in a Chinese population. Neonatology. 2013;104:127-32.

22. Piyasena C, Dhaliwal C, Russell H, et al. Prediction of severe retinopathy of prematurity using the WINROP algorithm in a birth cohort in South East Scotland. Arch Dis Child Fetal Neonatal Ed. 2014;99:F29-33.
23. Lucio KCDV, Bentlin MR, Augusto ACL, et al. The ROPScore as a screening algorithm for predicting retinopathy of prematurity in a Brazilian population. Clinics (Sao Paulo). 2018;73:e377.

24. Piermarocchi S, Bini S, Martini F, et al. Predictive algorithms for early detection of retinopathy of prematurity. Acta Ophthalmol. 2017;95:158-164.

25. Lundgren $\mathrm{P}$, Hård AL, Wilde $\AA$ et al. Implementing higher oxygen saturation targets reduced the impact of poor weight gain as a predictor for retinopathy of prematurity. Acta Paediatr. 2018;107:767-773.

\section{CLIPPINGS}

\section{Theme: Gastroenterology and Hepatology}

丹

Relationship between quantitative sonographic measurements and serum biochemical parameters in childhood obesity (Pediatr Gastroenterol Hepatol Nutr. 2021;24:470-82)

Childhood Obesity is global public health problem affecting not only the adolescents but also the young children, and its prevalence is increasing day by day. According to WHO, in 2016 over 340 million children and adolescents aged 5-19 were overweight or obese; 39 million under-five children were overweight or obese in 2020 . Obesity is closely related with the development of metabolic syndrome and excessive fat accumulation in hepatocytes leading to the development of nonalcoholic fatty liver disease (NAFLD) in children. In this study published from Turkey involving 174 overweight or obese children aged between 3-18 years (mean age of 10.6 year), the relationship between various indicators of obesity [e.g., BMI $z$ score, abdominal wall fat thickness, serum biochemical markers (AST, ALT, HDL, LDL, total cholesterol, Insulin and HOMAIR levels)] and sonographic measurements of fatty liver was assessed. Authors found a positive correlation between liverkidney echogenicity ratio (LKER) and serum transaminase and glucose levels in obese children. A positive correlation was also found between BMI $z$-score and abdominal wall fat thickness (AWFT) with fasting insulin level and HOMA-IR value. Authors concluded that due to the wide availability of the ultrasonography, it can be used as an effective tool in the management of the childhood obesity. Celiac disease in children: An association with drug-resistant epilepsy (Pediatr. Neurol 2021;120:12-17)

Seizures are one of the neurological manifestations in children with celiac disease. In the present study published from University of Utah, authors did a retrospective chart review to compare the children having epilepsy and celiac disease $(n=56)$ with 168 age- and sex-matched controls having only epilepsy, to analyze the effect of gluten-free diet on seizure burden. Study results showed that the children with celiac disease had a significantly higher percentage of drug-resistant epilepsy compared to control group, but comparable to the general population. Adherence to the gluten free diet along with the medications reduces the disease burden in children with celiac disease having drug resistance epilepsy.

Neutrophil to lymphocyte ratio and gastrointestinal involvement among Henoch Schonlein purpura patients: A systematic review and meta-Analysis $(\mathrm{J}$ Pediatr Gastroenterol Nutr. 2021;73:437-43)

Recently the Neutrophil to lymphocyte ratio has attracted much attention as a marker of systemic inflammation. A very simple investigation which is easily available and does not add to the cost of routine investigations and has been tested in various clinical conditions. In the current study the authors does the metaanalysis of the studies published on Henoch Schonlein purpura (HSP), to evaluate the difference in the neutrophil to lymphocyte ratio among the HSP patients presenting with and without gastrointestinal manifestations. They have analyzed 6 studies with low heterogeneity, and found that the patients of HSP with gastrointestinal involvement have higher neutrophil to lymphocyte ratio compared to those without gastrointestinal involvement [mean difference of 0.88 (95\% CI $0.55,1.22)$ ]. Authors concluded that the neutrophil to lymphocyte ratio in cases with HSP could serve as the marker of gastrointestinal involvement.

RaJesh Kumar Meena raj.mamc@gmail.com 